\title{
A study of two unequal-sized droplets undergoing oblique collision
}

Cite as: Phys. Fluids 33, 022110 (2021); https://doi.org/10.1063/5.0038734

Submitted: 25 November 2020 . Accepted: 20 January 2021 . Published Online: 26 February 2021

Gembali Sai Chaitanya, Kirti Chandra Sahu, and (D) Gautam Biswas
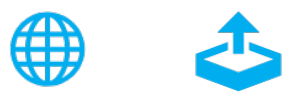

Export Citation

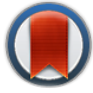

View Online

CrossMark

\section{ARTICLES YOU MAY BE INTERESTED IN}

A comparison of bioinspired slippery and superhydrophobic surfaces: Micro-droplet impact Physics of Fluids 33, 022105 (2021); https://doi.org/10.1063/5.0035556

Ferrohydrodynamics governed evaporation phenomenology of sessile droplets

Physics of Fluids 33, 022006 (2021); https://doi.org/10.1063/5.0040712

Solute induced jittery motion of self-propelled droplets

Physics of Fluids 33, 022103 (2021); https://doi.org/10.1063/5.0038716

Physics of Fluids SPECIAL TOPIC: Tribute to Frank M. White on his 88th Anniversary 


\title{
A study of two unequal-sized droplets undergoing oblique collision
}

\author{
Cite as: Phys. Fluids 33, 022110 (2021); doi: 10.1063/5.0038734 \\ Submitted: 25 November 2020 - Accepted: 20 January 2021 . \\ Published Online: 26 February 2021
}

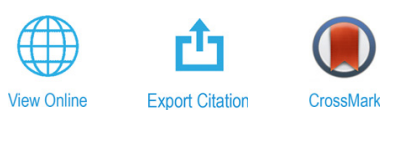

\section{Gembali Sai Chaitanya,' (D) Kirti Chandra Sahu, ${ }^{2}$ (D) and Gautam Biswas ${ }^{1, a)}$ (D)}

\section{AFFILIATIONS}

'Department of Mechanical Engineering, Indian Institute of Technology Kanpur, Kanpur 208016, Uttar Pradesh, India

${ }^{2}$ Department of Chemical Engineering, Indian Institute of Technology Hyderabad, Sangareddy 502285, Telengana, India

a) Author to whom correspondence should be addressed: gtm@iitk.ac.in

\begin{abstract}
The oblique collision of two unequal-sized liquid droplets in a gaseous environment is investigated numerically. It is found that the asymmetry in the flow field arising due to unequal-sized droplets and oblique collision greatly alters the collision outcomes observed in the case of the head-on collision of identical droplets. Our results reveal that permanent coalescence occurs at intermediate collision angles, but head-on and large-angle collisions result in reflexive separation and stretching separation, respectively. Moreover, we found that the end-pinching mechanism is operational in the case of head-on collision, and the capillary wave instability is responsible for the ligament breakup for large collision angles. It is also observed that the droplets coalesce permanently for low velocity ratios and high radius ratios, but for high velocity ratios and low radius ratios, the droplets coalesce temporarily and then split again. By conducting a large number of numerical simulations, the collision outcomes and the boundary separating them are plotted on $R_{r}-W e$ and $\theta-W e$ planes, where We, $R_{r}$, and $\theta$ represent the Weber number, radius ratio of droplets, and collision angle, respectively.
\end{abstract}

Published under license by AIP Publishing. https://doi.org/10.1063/5.0038734

\section{INTRODUCTION}

The phenomenon of the collision of liquid droplets has been extensively investigated because of its relevance in many industrial applications, ${ }^{1,2}$ such as nuclear fusion, sprays, ink-jet printing, and internal combustion processes, and also in meteorological applications, such as the formation of raindrops. ${ }^{3-7}$ Several researchers have also investigated the coalescence dynamics of a liquid droplet on a flat liquid pool (see, e.g., Refs. 8-14). Different collision outcomes have been observed when two equal-sized droplets undergo collision depending on the Weber number. ${ }^{3}$ During the collision of two droplets, high pressure develops in the gap, causing the droplets to flatten, and the thin film of the surrounding fluid squeezes out. The droplets undergo coalescence if the gap between them reduces to a dimension comparable to that of molecular interaction, usually on the order of $10^{2} \AA$; otherwise, they exhibit bouncing behavior. ${ }^{15}$ Whereas coalescence of a droplet on a flat liquid surface produces a satellite droplet (which eventually merges in the liquid pool via the well-known coalescence cascading process), the collision of two equal-sized droplets does not exhibit such behavior. $^{16}$

It was believed that the collision outcomes depend primarily on the Weber number, but Qian and Law ${ }^{17}$ demonstrated experimentally that the properties of the surrounding medium and the viscosity of the liquid also play an important role. Eggers et al. ${ }^{18}$ also theoretically showed that the coalescence process is significantly influenced by the viscosity of the surrounding fluid. By considering both head-on and oblique collisions of identical water and hydrocarbon droplets at different surrounding pressures, Qian and $\mathrm{Law}^{17}$ reported five distinct outcomes, namely, (i) coalescence after minor deformation, (ii) bouncing, (iii) coalescence after substantial deformation, (iv) coalescence followed by separation for near head-on collisions, and (v) coalescence followed by separation for off-center/oblique collisions. However, two equal-sized water droplets in air at $1 \mathrm{~atm}$ only exhibit coalescence after minor deformation (outcome "i") and coalescence followed by separation (outcomes "iv" and "v"). The transition between the coalescence and separation regimes has also been identified. Subsequently, for a wide range of Weber numbers, Pan et al. ${ }^{19}$ studied the head-on collision of two identical droplets experimentally and numerically. The experiments were conducted using a time-resolved microphotographic technique similar to that of Qian and Law, ${ }^{17}$ and the numerical simulations were performed using a front tracking method. ${ }^{20}$ They showed that the merging instant can be evaluated computationally by the use of an augmented van der Waals force and the associated Hamaker constant extracted empirically from experimental observations in both 
soft and hard collisions, which correspond to the droplets undergoing collision with minor and significant deformations, respectively. Nobari et al. ${ }^{21}$ also studied the head-on collision of equal-sized droplets using a front tracking/finite difference technique and identified the boundaries between coalescing and separating collisions on the Reynolds number and Weber number plane.

All of the studies discussed above considered the collision of equal-sized droplets. The collision dynamics of unequal-sized droplets have been attracting increasing attention (see, e.g., Refs. 16 and 22). Zhang et al. ${ }^{16}$ studied experimentally the coalescence dynamics of a small droplet falling on a larger droplet and found that the formation of satellite droplet occurs when the diameter ratio is greater than 1.55, which increases with increasing the Ohnesorge number $\left(O h=\mu_{l} / \sqrt{\rho_{l} \sigma R_{s}}\right)$, where $\mu_{l}$ is the dynamic viscosity of the liquid and $R_{s}$ is the radius of the small droplet). Kumar et al. ${ }^{23}$ investigated experimentally the coalescence dynamics of a freely falling ethanol droplet on a sessile droplet of the same fluid using a highspeed imaging system. They showed regime maps in terms of the partial coalescence and spreading behaviors on the plane of the Weber number and the volume ratio of the sessile and the impacting droplets. The critical value of the size ratio of the droplets for the formation of a satellite droplet was found to be consistent with that of Zhang et al. ${ }^{16}$ Nikolopoulos et al. ${ }^{24}$ focused on the splashing regime by impacting a droplet from a height. Tang et al. ${ }^{22}$ investigated theoretically and experimentally the dynamics of the head-on collision of unequal-sized hydrocarbon and water droplets, and identified the regions associated with bouncing, permanent coalescence, and separation after coalescence in the parameter space of the size ratio and the collision Weber number. Deka et al. ${ }^{25}$ studied numerically the head-on collision of unequal-sized droplets and reported that the collision leads to partial coalescence of very small daughter droplets even for a diameter ratio equal to 1.2. Cong et $a l .{ }^{26}$ investigated the collision dynamics of unequal-sized droplets at high Weber numbers. By conducting axisymmetric simulations, Goyal et al. ${ }^{27}$ also investigated the head-on collision of two unequal-sized droplets.

As the above brief review shows, apart from the experimental study of Qian and Law, ${ }^{17}$ who studied the collision of two equalsized droplets, the work carried out on this subject has so far been for a head-on configuration. Oblique collision is expected to alter the flow field inside and outside the droplets, which can in turn influence the collision outcomes. ${ }^{17}$ Since the flow field for small droplets undergoing collision is difficult to obtain experimentally, it is necessary to study the collision dynamics of two unequal-sized droplets by performing numerical simulations, which is the focus of this work. We used a coupled-level-set-volume-of-fluid (CLSVOF) approach to study the dynamics of two unequal-sized droplets, approaching at an angle with different velocities. The numerical solver was validated against previous experimental and numerical results for the head-on collision of two identical droplets. A parametric study was conducted by varying the collision angle $(\theta)$, radius ratio $\left(R_{r}\right)$, and velocity ratio $\left(V_{r}\right)$, and the results were analyzed in terms of the velocity field. The remainder of the paper is organized as follows: In Sec. II, the problem is formulated, and the grid convergence test and validation of the solver are conducted. The results are discussed in Sec. III, and concluding remarks are given in Sec. IV.

\section{FORMULATION}

The collision dynamics of two unequal-sized initially spherical droplets of radii $R_{1}$ and $R_{2}$ approaching with velocities $V_{1}$ and $V_{2}$ and undergoing oblique collision is investigated numerically in the framework of the coupled-level-set-volume-of-fluid (CLSVOF) approach. The oblique collision angle (i.e., the angle created by the collision direction and the line joining the initial centers of the droplets) is $\theta$, such that $\theta=0$ represents the limiting case of the head-on collision. A schematic diagram showing the initial configuration of the droplets is presented in Fig. 1. A two-dimensional coordinate system $(x, y)$ is used to model the flow dynamics. The initial separation distance between the center of the droplets is $\beta=2.5 R_{L}$, where $R_{L}$ is the radius of the bigger droplet, such that $\beta \cos (\theta)$ and $\beta \sin (\theta)$ are the projections of the separation distance along the $x$ - and $y$ - axes, respectively. The initial center clearance between droplets, $h_{0}$, is $\beta-R_{1}-R_{2}$. The dynamic viscosity and density of the liquid droplets and surrounding gaseous medium are $\left(\mu_{l}, \rho_{l}\right)$ and $\left(\mu_{g}, \rho_{g}\right)$, respectively, and $\sigma$ represents the interfacial tension of the fluid pairs. The droplets were liquid tetradecane and the surrounding medium was considered to be either air or nitrogen gas in the various cases examined in this study. The fluids were assumed to be Newtonian and incompressible. The properties of the fluids are given in Table I. These fluids were chosen to facilitate the comparison with an earlier experimental study. ${ }^{19}$ The size of the computational domain was considered to be large enough that the boundary had a negligible effect on the collision phenomenon.

\section{A. Governing equations}

The collision dynamics of two unequal-sized droplets are governed by the continuity and Navier-Stokes equations, which are given by

$$
\begin{gathered}
\nabla \cdot \vec{V}=0, \\
\rho(\phi)\left[\frac{\partial \vec{V}}{\partial t}+\vec{V} \cdot \nabla \vec{V}\right]=-\nabla P+\nabla \cdot\left[\mu(\phi)\left(\nabla \vec{V}+\nabla \vec{V}^{T}\right)\right] \\
+\sigma \kappa(\phi) \hat{n} \delta_{\epsilon}(\phi),
\end{gathered}
$$

where $\vec{V}$ is the velocity field, wherein $u$ and $v$ represent the component of velocity in the $x$ and $y$ directions, respectively; $\rho(\phi)$ is the density;

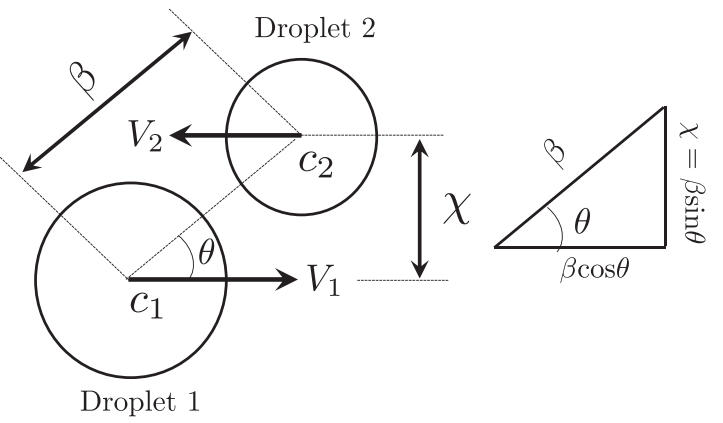

FIG. 1. Schematic diagram showing two droplets of radii $R_{1}$ and $R_{2}$ colliding with velocities $V_{1}$ and $V_{2}$ at an oblique angle $\theta . \chi$ is the projection distance and $\beta=$ $2.5 R_{L}$ is the initial separation distance between the droplets, where $R_{L}$ is radius of the bigger droplet. The density and dynamic viscosity of the droplets and the surrounding medium are $\rho_{\mathrm{k}}, \mu_{\mathrm{f}}$ and $\rho_{\mathbf{g}}, \mu_{\mathrm{g}}$, respectively. 
TABLE I. Fluid properties of the liquid and gaseous phases considered at $1 \mathrm{~atm}$ and $25^{\circ} \mathrm{C}$. The values of the surface tension of tetradecane with respect to air and nitrogen gas are $0.027 \mathrm{~N} / \mathrm{m}$ and $0.026 \mathrm{~N} / \mathrm{m}$, respectively.

\begin{tabular}{lcc}
\hline \hline Fluids & Density $\left(\mathrm{kg} / \mathrm{m}^{3}\right)$ & Viscosity $(\mathrm{Pas})$ \\
\hline Tetradecane (droplets) & 758 & $2.128 \times 10^{-3}$ \\
Air (surrounding) & 1.225 & $1.78 \times 10^{-5}$ \\
Nitrogen (surrounding) & 1.138 & $1.787 \times 10^{-5}$ \\
\hline \hline
\end{tabular}

$\hat{n}(=\nabla \phi / \mid \nabla \phi)$ is the outward pointing unit normal vector at the interface; $\kappa(=-\nabla \cdot \hat{n})$ is the mean curvature of the interface; $\phi$ represents the level-set function; $\delta_{\epsilon}(\phi)$ is a Dirac delta distribution function; and $t$ denotes time. The surface tension force is included as a body force term in Eq. (2) by using the continuum surface force (CSF) model. ${ }^{28}$ In present study, we considered small droplets (radius $100 \mu \mathrm{m}-240 \mu \mathrm{m}$ ), so the Bond number $\rho_{g} g\left(2 R_{1}\right)^{2} /$ $\sigma<10^{-4}$. Therefore, the effect of gravity force is neglected in the present study.

The interfacial dynamics are modeled using the level-set function $(\phi)$ and the volume fraction of the liquid phase $(F)$ by solving the following advection equations:

$$
\begin{aligned}
& \frac{\partial \phi}{\partial t}+\vec{V} \cdot \nabla \phi=0, \\
& \frac{\partial F}{\partial t}+\vec{V} \cdot \nabla F=0,
\end{aligned}
$$

where $F=0$ for the gaseous phase and $F=1$ for the liquid phase. For a cell containing both the liquid and gaseous phases, the value of $F$ lies between 0 and 1 . Similarly, the values of the level-set function $(\phi)$ for the gaseous and liquid phases are $\epsilon$ and $-\epsilon$, respectively, and 0 at the interface separating the fluids. Here, $\epsilon$ is the numerical thickness of the interface. In the present study, we used $\epsilon=1.5 \Delta$, where $\Delta$ is the grid size.

The density and dynamic viscosity of both the phases are modeled using a Heaviside function, $H_{\epsilon}(\phi)$, as

$$
\begin{gathered}
\rho(\phi)=\rho_{l} H_{\epsilon}(\phi)+\rho_{g}\left(1-H_{\epsilon}(\phi)\right), \\
\mu(\phi)=\mu_{l} H_{\epsilon}(\phi)+\mu_{g}\left(1-H_{\epsilon}(\phi)\right),
\end{gathered}
$$

where the Heaviside function $H(\phi)$ is given by

$$
H_{\epsilon}(\phi)= \begin{cases}1, & \text { if } \phi>\epsilon \\ \frac{1}{2}+\frac{\phi}{2 \epsilon}+\frac{1}{2 \pi}\left\{\sin \left(\frac{\pi \phi}{\epsilon}\right)\right\}, & \text { if }|\phi| \leq \epsilon \\ 0, & \text { if } \phi<-\epsilon .\end{cases}
$$

The regularized Dirac delta distribution function, $\delta_{\epsilon}(\phi)$, is given by

$$
\delta_{\epsilon}(\phi)=\left\{\begin{array}{l}
\frac{1}{2 \epsilon}+\frac{1}{2 \epsilon} \cos \left(\frac{\pi \phi}{\epsilon}\right), \text { if }|\phi| \leq \epsilon \\
0, \quad \text { otherwise, }
\end{array}\right.
$$

which is used to model the surface tension force term that acts only in the interfacial layer, i.e., with $|\phi| \leq \epsilon$.

\section{B. Numerical method and boundary conditions}

A coupled-level-set-volume-of-fluid (CLSVOF) method $^{29}$ based on a staggered grid formulation is used. The advantage of this method is that it improves the conservation mass while maintaining a sharp interface. The numerical method used in this work is the same as that used in our previous studies on the coalescence of multiple drops impacting on the free surface of a liquid pool ${ }^{13}$ and coalescence of unequal-sized droplets resting on each other. ${ }^{25}$ However, for the sake of completeness, below we briefly discuss the numerical method used in this study. The computational domain (Fig. 1) is discretized using a uniform grid size $(\Delta)$ in the $x$ and $y$ directions. The marker and cell (MAC) algorithm is employed to solve the single set of governing equations, describing both phases in a staggered grid arrangement, ${ }^{30}$ such that the vector quantities (e.g., the velocity) are defined at the center of the cell faces normal to them, and the scalar variables (e.g., the pressure, volume fraction and level set function) are defined at the cell centers. A finite-difference method is used to discretize the governing equations [Eqs. (1)-(4)]. The convective terms in Eqs. (2) and (3) are discretized using the higher-order essentially non-oscillatory (ENO) scheme, ${ }^{31,32}$ and the remaining space derivatives are discretized using a central difference scheme. The second-order conservative operator split advection scheme $\mathrm{e}^{33}$ is used for discretization of conservative form of the volume fraction advection equation [Eq. (4)], and the conservation of $F$ is maintained by employing an implicit scheme in the first sweeping direction and an explicit scheme in the second sweeping direction. The discretized form of the momentum equations [Eq. (2)] is solved explicitly for the known volume fraction field $F_{n}$ and the level set function $\phi_{n}$, where $n$ is the time iteration level. This step gives the provisional velocity field (non-divergence free) at a time iteration level $n$. The compliance of the continuity equation is achieved by solving the corresponding pressure correction equation using the Hypre-multigrid solver. After achieving a divergence-free velocity field, the converged solution is obtained at the next time level $(n+1)$. Then, using the updated velocity field, the advection equations of the volume fraction and the level set function are solved to obtain the new volume fraction field $F_{(n+1)}$ and the level set function $\phi_{(n+1)}$. At each time step after finding the updated volume fraction $F_{(n+1)}$ and level set function $\phi_{(n+1)}$, the level set function is reinitialized to the exact signed normal distance from the reconstructed interface by coupling the level set function with the volume fraction. ${ }^{34-36}$ In this work, the timestepping procedure is based on an explicit method to maintain the stability of the solution. During the computations, the time step is chosen to satisfy the Courant-Friedrichs-Lewy (CFL), capillary, and viscous time conditions. The Neumann boundary conditions are used for all the boundaries of the computational domain.

It should be noted that the numerical model is based on continuum mechanics, whereas interfacial dynamics would necessarily incorporate intermolecular forces and rarified flow during the coalescence event. The minimum grid size used in the simulation is around $2 \mu \mathrm{m}$, which is too large to capture a real gas film, which is on the order of $10^{2} \AA$. As in practice, it is not possible to resolve the real film thickness; in the continuum mechanics framework, this can be handled by specifying the instant of rupture/rebound of colliding interfaces as an input to the computational model. ${ }^{19}$ In describing the results presented in this study, we not only provide the actual dimensional parameters used, but also give the values of the associated dimensionless numbers, namely, the Reynolds number $\left(\operatorname{Re}\left(\equiv 2 \rho_{\ddagger} V_{1} R_{1} / \mu_{\sharp}\right)\right)$, the Weber 
(a)

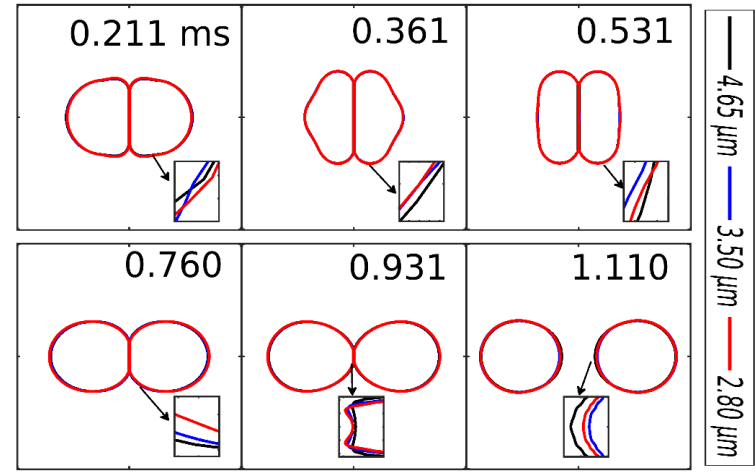

(b)

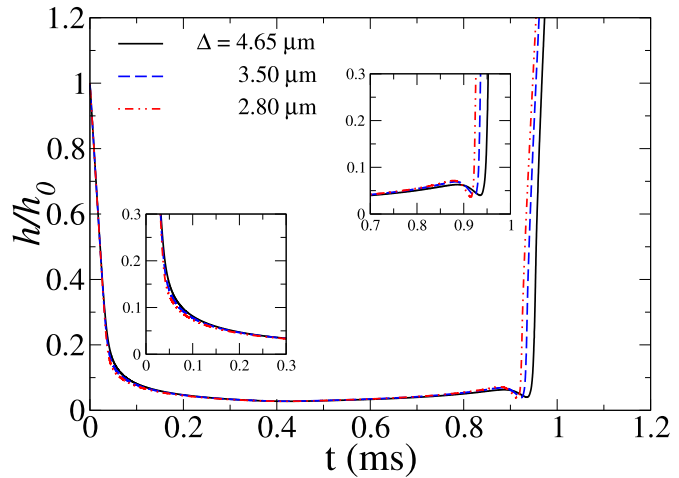

FIG. 2. Grid convergence test performed using $\Delta=4.65,3.50$, and $2.80 \mu \mathrm{m}$ for the collision of two tetradecane droplets in air. Temporal evolution of (a) the deformation and coalescence dynamics of the droplets [time in milliseconds (ms) is written at the top right corner of each panel] and (b) normalized center-clearance between droplets, $h / h_{0}$. The rest of the dimensional parameters are $\theta=0^{\circ}, R_{1}=R_{2}=167.6 \mu \mathrm{m}, V_{1}=V_{2}=0.496 \mathrm{~m} / \mathrm{s}$. The corresponding dimensionless numbers are $W e=2.32$, Re $=59.23$, $V_{r}=1$, and $R_{r}=1$. The insets in (b) represent the magnified views for $0 \leq t \leq 0.3 \mathrm{~ms}$ and $0.7 \leq t \leq 1 \mathrm{~ms}$.

number $\left(W e\left(\equiv 2 \rho_{\mathrm{y}} V_{1}^{2} R_{1} / \sigma\right)\right)$, the velocity ratio $\left(V_{r}\left(\equiv V_{2} / V_{1}\right)\right)$, the radius ratio $\left(R_{r}\left(\equiv R_{2} / R_{1}\right)\right)$, the density ratio $\left(\rho_{r}\left(\equiv \rho_{\mathrm{g}} / \rho_{\mathrm{Y}}\right)\right)$, the viscosity ratio $\left(\mu_{r}\left(\equiv \mu_{\mathrm{g}} / \mu_{\mathrm{f}}\right)\right)$, and the dimensionless time $t^{*}$ $=t\left(V_{1} / 2 R_{1}\right)$. In the present study, in order to vary the velocity and radius ratios, we have fixed the velocity and radius of droplet 1 and varied the radius and velocity of droplet 2 .

\section{Validation}

To validate the current numerical solver, we have conducted a grid convergence test and compared the results obtained using the current solver with the experimental results of Pan et al. ${ }^{19}$ In Figs. 2(a) and 2(b), we investigate the effect of varying the grid size, $\Delta$, on the temporal evolution of the coalescence dynamics and temporal variation of the normalized center-clearance distance between the droplets, $h / h_{0}$, respectively. The parameters considered to generate these plots are $\theta=0^{\circ}, R_{1}=R_{2}=167.6 \mu \mathrm{m}$, and $V_{1}=V_{2}=0.496 \mathrm{~m} / \mathrm{s}$, which represent the head-on collision and subsequent bouncing of two equal-sized tetradecane droplets approaching at the same velocity in air (for $W e=2.32, R e=59.23$ ). The results in Fig. 2(a) reveal that the droplets exhibit qualitatively identical shape deformation for different grids. Figure 2(b) also shows that good quantitative agreement is achieved in the temporal variations of the normalized center clearance between droplets $\left(h / h_{0}\right)$ obtained using different grid sizes. However, it can be seen in Fig. 2(b) that after bouncing $(t>0.9)$, the coarsest grid $4.65 \mu \mathrm{m}\left(\Delta=R_{1} / 36\right)$ deviates slightly from the results obtained using the finer grid sizes (namely, $3.50 \mu \mathrm{m}\left(\Delta=R_{1} / 48\right)$ and $2.80 \mu \mathrm{m}$ $\left.\left(\Delta=R_{1} / 60\right)\right)$. However, the results obtained using $3.50 \mu \mathrm{m}$ $\left(\Delta=R_{1} / 48\right)$ and $2.80 \mu \mathrm{m}\left(\Delta=R_{1} / 60\right)$ are quite close. In view of this, we adopt a grid size of $3.50 \mu \mathrm{m}\left(\Delta=R_{1} / 48\right)$ to generate the remainder of the results presented in this study.

Figure 3 shows the comparison of the normalized kinetic energy $\left(E_{k} / E_{K 0}\right)$ and surface energy $\left(E_{s} / E_{s 0}\right)$ obtained from the present simulation and the numerical simulation by Pan et al. ${ }^{19}$ Here, $E_{k}$ $=\frac{1}{2} \sum_{i=1}^{N} F_{i} \rho_{l}\left(u_{i}^{2}+v_{i}^{2}\right) v_{\text {cell }}$ and $E_{s}=\sigma \sum A_{s}$, wherein $v_{\text {cell }}$ is the volume of the computational cell, $F_{i}$ is the volume fraction, and $u_{i}$ and $v_{i}$ are the velocity components, $N$ represents the number of computational cells, and $\sum A_{s}$ represents the summation of surface area of all the interfacial cells. $E_{k 0}$ and $E_{s 0}$ are the initial values of $E_{k}$ and $E_{s}$. A front tracking method in an axisymmetric domain was used by Pan et al. ${ }^{19}$ in their numerical simulations. It can be seen in Fig. 3 that the comparison is indeed satisfactory. Next, in Figs. 4 and 5, we present the dynamics of two equal-sized tetradecane droplets in air undergoing head-on collision and compare them with the numerical and experimental results of Pan et al. ${ }^{19}$ Figures 4 and 5 depict bouncing and coalescence behaviors, respectively. It can be seen that the droplets undergo significant shape deformation. These types of collision were termed hard collision by Pan et al. ${ }^{19}$ We also compared the bouncing and coalescence behaviors of droplets with slight deformation (not shown), which were termed soft collisions by Pan et al. ${ }^{19}$ They also found that for soft and hard collisions the transition from coalescence and bouncing behaviors occurs at $W e=0.565$ and 3.075, respectively. It can be observed in Figs. 4 and 5 that the present numerical solver accurately predicts the shape deformation during the

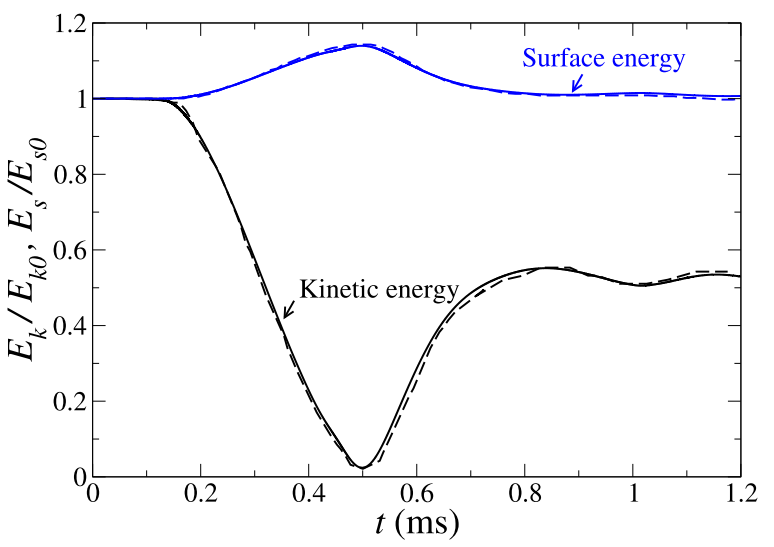

FIG. 3. Comparison of the normalized droplet kinetic energy $\left(E_{k} / E_{k 0}\right)$ and surface energy $\left(E_{s} / E_{s 0}\right)$ obtained from the present numerical simulation (solid line) and the numerical simulation by Pan et al. ${ }^{19}$ (dashed line). The parameters are the same as those used to generate Fig. 2. 
<smiles>C1CCCCCCC1</smiles>

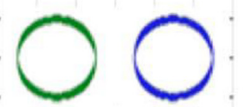

$0.300 \mathrm{~ms}$<smiles>C1CCC2CCCCC2CC1</smiles><smiles>C1CC[C@H]2CCCC[C@H]2CC1</smiles>

$\mathbf{0 . 5 0 0}$<smiles>C1CCC2CCCC2CC1</smiles><smiles>C1CCC2CCCCC2CC1</smiles><smiles>C1CCCC2(CC1)CCCCCC2</smiles>

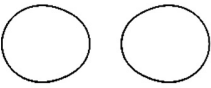

(a)

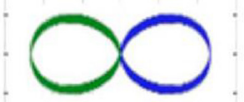

1.285

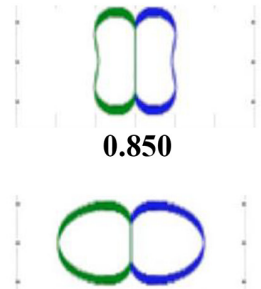

1.100

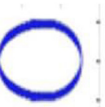

1.400

(b)
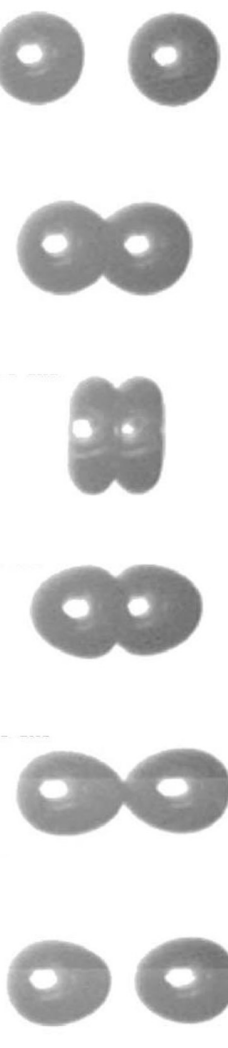

(c)

FIG. 4. Temporal evolution of two tetradecane droplets in air for $\theta=0^{\circ}, R_{1}=R_{2}$ $=167.6 \mu \mathrm{m}, V_{1}=V_{2}=0.496 \mathrm{~m} / \mathrm{s}$. The corresponding dimensionless numbers are $W e=2.32, R e=59.23, V_{r}=1$, and $R_{r}=1$. Results obtained from (a) the present numerical simulation, (b) numerical simulation by Pan et al., ${ }^{19}$ and (b) experimental results of Pan et al. ${ }^{19}$ Here, the droplets undergo bouncing after significant deformation (hard collision).

bouncing and coalescence of droplets. We also found that the rupture and rebound times for bouncing and coalescing droplets are in good agreement with those reported by Pan et al. ${ }^{19}$

\section{RESULTS AND DISCUSSION}

In this section, we discuss our numerical results. Particular attention is given to the effect of varying the collision angle $(\theta)$, velocity ratio $\left(V_{r}\right)$ and radius ratio $\left(R_{r}\right)$ on the collision dynamics of two droplets since the head-on collision and identical droplets have been well studied. We identified different collision outcomes for two identical and unequal-sized hydrocarbon droplets undergoing oblique collision by performing a large number of numerical simulations and contrasted the results with the dynamics observed in the case of head-on collision. As discussed in Sec. I, when two droplets collide in a headon configuration, mainly three collision outcomes are observed, depending on the impact velocity and fluid properties of the droplets and the surrounding environment, namely, coalescence, bouncing, and separation. ${ }^{17,21}$ In the case of collisions resulting in coalescence, the surrounding fluid separating the droplets is squeezed out quickly
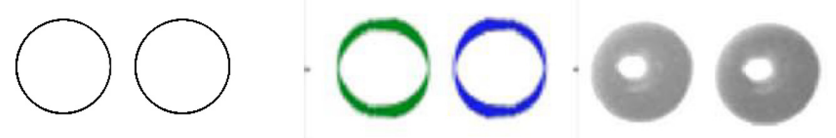

$0.050 \mathrm{~ms}$
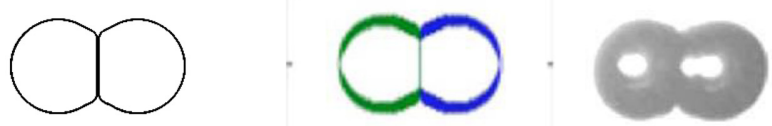

0.180
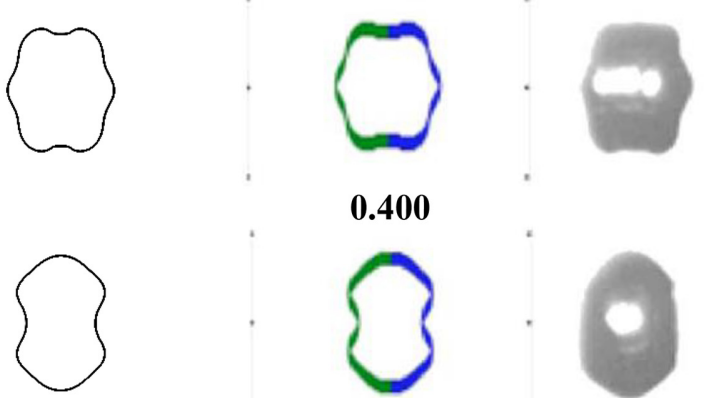

0.400
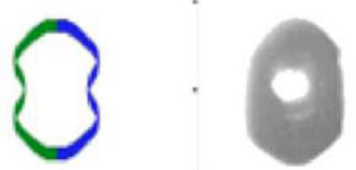

0.500
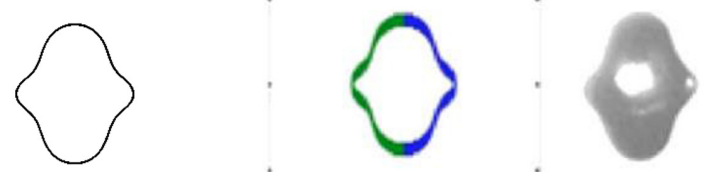

0.580
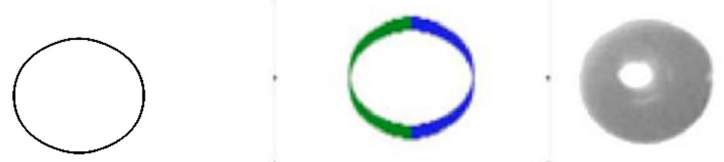

0.900

(a)

(b)

(c)

FIG. 5. Temporal evolution of two tetradecane droplets in air for $\theta=0^{\circ}, R_{1}=R_{2}$ $=169.7 \mu \mathrm{m}, V_{1}=V_{2}=0.596 \mathrm{~m} / \mathrm{s}$. The corresponding dimensionless numbers are $W e=3.4, R e=72.1, V_{r}=1$, and $R_{r}=1$. Results obtained from (a) the present numerical simulation, (b) numerical simulation by Pan et al., ${ }^{19}$ and (b) experimental results of Pan et al. ${ }^{19}$ Here, the droplets undergo bouncing after significant deformation (hard collision).

and the droplets coalesce permanently. The merged mass undergoes shape deformation and oscillations due to the competition between the kinetic energy and surface energy. As time progresses, these oscillations become damped as the kinetic energy associated with fluid motion in the droplet dissipates due to viscous dissipation, and eventually the merged fluid mass acquires a spherical shape. In the case of bouncing, the fluid layer becomes trapped between the droplets, which prevents the droplets from coalescing. The droplets continue to deform until the internal kinetic energy reaches a minimum and high pressure develops in the region separating the droplets. At this stage, the flow within the droplet redirects sharply in the directions opposite 


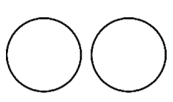

$0.00 \mathrm{~ms}$

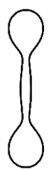

0.223

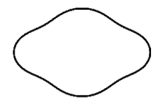

0.365

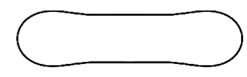

0.514

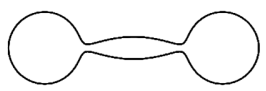

0.843
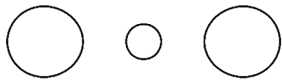

1.192

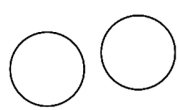

0.00

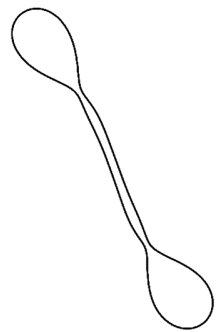

0.751

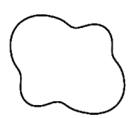

1.125

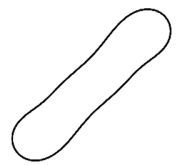

1.246

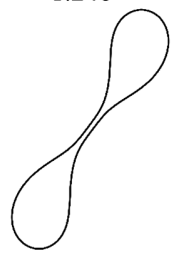

1.597

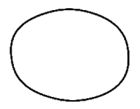

2.327<smiles>C1CCCCCCCC1</smiles>

0.00

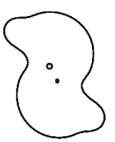

0.080

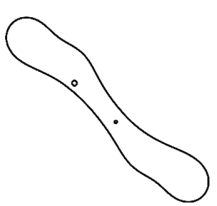

0.180

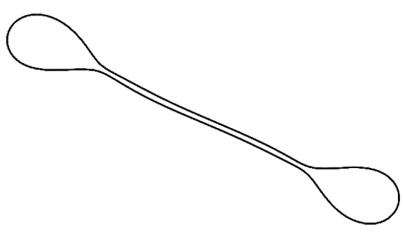

0.510
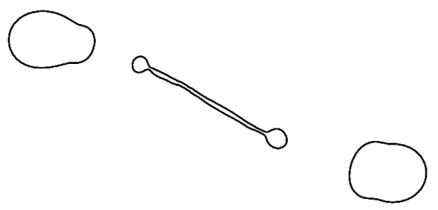

0.663

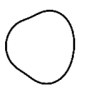

0<smiles>[O]</smiles><smiles>C1CCCCC1</smiles>

0.733

(c)
FIG. 6. Effect of the angle of collision $(\theta)$ on the coalescence dynamics of two tetradecane droplets in nitrogen at $1 \mathrm{~atm}$ (a) $\theta=0^{\circ}, \quad$ (b) $\theta=10.37^{\circ}$, and (c) $\theta=28.68^{\circ}$. The other dimensional parameters are $R_{1}=R_{2}=100 \mu \mathrm{m}$, $V_{1}=V_{2}=1.656 \mathrm{~m} / \mathrm{s}$. The corresponding dimensionless numbers are $W e=16$, $R e=118.01, V_{r}=1$, and $R_{r}=1$.

(a)

(b)

to the impinging directions and the droplets gradually start to gain kinetic energy, and as a result the gap between them widens and finally they regain their spherical shapes due to surface tension. The rebounding velocities, however, are smaller than the impacting velocities of the droplets because some amount of energy is lost via the viscous dissipation. In the case of separation, the droplets coalesce temporarily and then split again.

In Fig. 6, we investigate the effect of varying the collision angle
on the collision dynamics of two equal-sized tetradecane droplets of radii $R_{1}=R_{2}=100 \mu \mathrm{m}$, impacting at the same velocities, $V_{1}$ $=V_{2}=1.656 \mathrm{~m} / \mathrm{s}$ in a nitrogen environment at $1 \mathrm{~atm}$. The values of the corresponding dimensionless numbers are $W e=16, R e=118.01$, $V_{r}=1$ and $R_{r}=1$. The temporal evolutions of the droplets for $\theta=0^{\circ}$ (head-on collision), $\theta=10.37^{\circ}$, and $\theta=28.68^{\circ}$ are presented in 

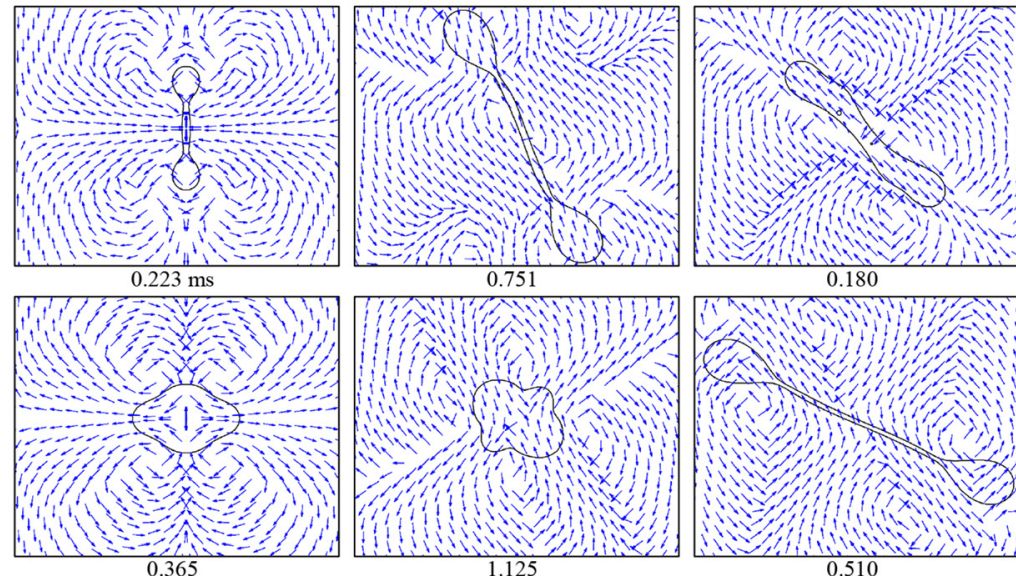

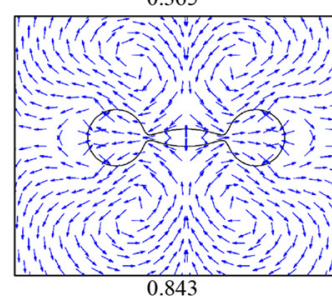

(i)

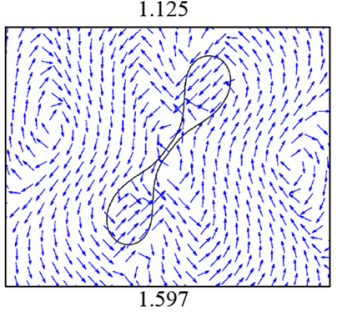

(ii)

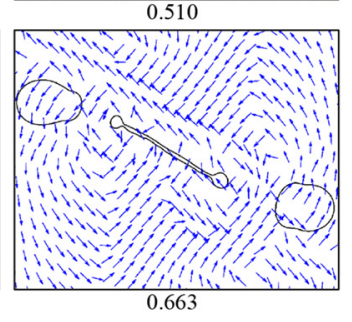

(iii)

(a)

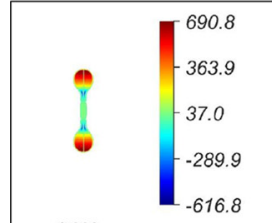

$0.223 \mathrm{~ms}$
-709.8
-475.9
-241.9
8.0

0.365

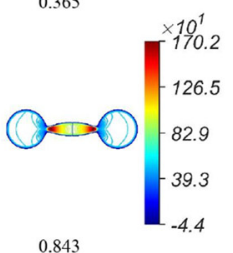

(i)

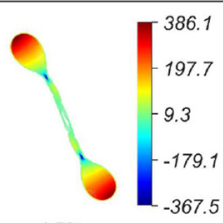

0.751

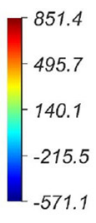

1.125

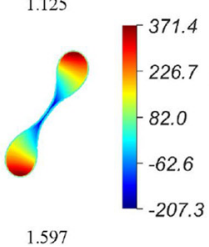

(ii)

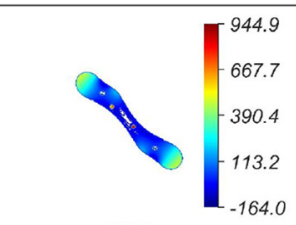

0.180

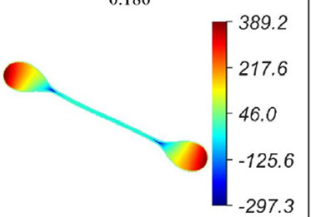

0.510

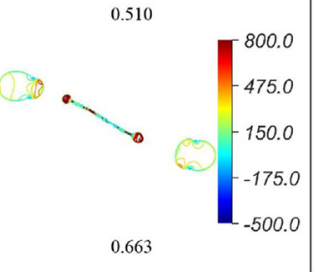

(iii)

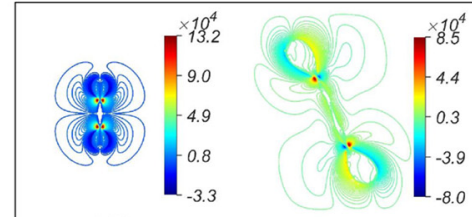

$0.223 \mathrm{~ms}$

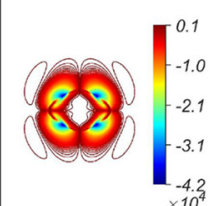

0.365

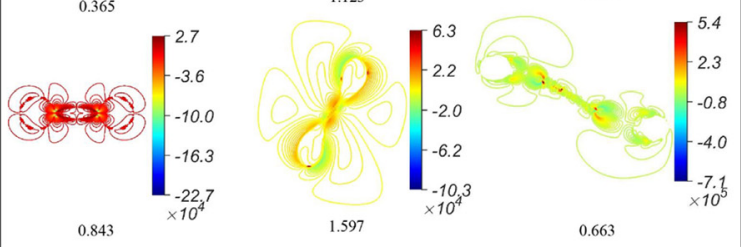

(i)

(ii)

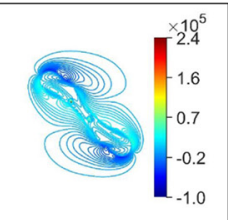

0.180

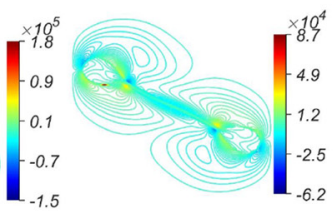

0.510

(iii)

(b)

(c)

FIG. 7. Temporal evolution of (a) flow field, (b) pressure distribution, and (c) vorticity $(\partial v / \partial x-\partial u / \partial y)$ field for different values of angle of collision in the case of collision of two tetradecane droplets in nitrogen at $1 \mathrm{~atm}$. In each panel, (i) $\theta=0^{\circ}$, (ii) $\theta=10.37^{\circ}$, and (iii) $\theta=28.68^{\circ}$. The other dimensional parameters are $R_{1}=R_{2}=100 \mu \mathrm{m}$, $V_{1}=V_{2}=1.656 \mathrm{~m} / \mathrm{s}$. The corresponding dimensionless numbers are $W e=16, R e=118.01, V_{r}=1$, and $R_{r}=1$. 
Figs. 6(a)-6(c), respectively. The corresponding flow fields for $\theta=0^{\circ}$ (head-on collision), $\theta=10.37^{\circ}$, and $\theta=28.68^{\circ}$ are depicted in Figs. 7(a-i)-7(a-iii), respectively.

In Fig. 6(a), it can be seen that just after the collision the droplets exhibit an expansion, resulting in an elongated disk-shaped droplet (perpendicular to the impacting direction). At this stage, the surrounding gas moves inwards into the rim part of the disk shape [see $t=0.223 \mathrm{~ms}$ in Fig. $7(\mathrm{a}-\mathrm{i})]$. The inward flow pushing the liquid into the rim part of the disk is prevented by the change in the surface curvature that creates a difference in pressure between the fluid at the rim and the central portion of the droplet ${ }^{3}$ [see, Fig. 7(b)]. The deformed droplet at $t=0.223 \mathrm{~ms}$ possesses extra residual surface energy compared with the total surface energy of both parent droplets. This is because the kinetic energy associated with internal fluid motion in the combined droplet mass is converted into surface energy during deformation, and some of it is also dissipated due to viscosity (viscous dissipation). The decrease in the kinetic energy of flow and the reflexive action by the liquid surface due to the surface tension tries to bring the droplet to a spherical shape, but inertia elongates it in the direction of impact $(t=0.365 \mathrm{~ms})$ to form a stretched liquid cylinder at $t=0.514 \mathrm{~ms}$. The flow field at $t=0.365 \mathrm{~ms}$ [Fig. 7(a-i)] clearly shows four symmetrical recirculating regions in surrounding gas and the internal flow is directed toward the center of the droplet [Fig. 7(a-i)]. At $t=0.514 \mathrm{~ms}$, the fluid at the rounded ends of the liquid cylinder possesses a higher internal pressure than the remainder of the cylinder owing to its larger curvature as compared with the midsection of the cylinder, which in turn creates a local flow that pulls the ends toward the center. Subsequently, the liquid cylinder evolves into a dumbbell shape because of the outwardly directed internal motion $(t=0.843 \mathrm{~ms})$. If the kinetic energy associated with the internal fluid motion within the bulbous ends is not sufficient to split the droplet mass apart, then surface tension will pull back the ends of the dumbbell-shaped droplet. However, if the outwardly directed motion is sufficiently large, then the end droplets will detach from the ligament and the remaining fluid in the ligament contracts to form satellite droplets, as can be seen at $t=1.192 \mathrm{~ms}$ in Fig. 6(a). This collision outcome is termed "reflexive separation" in the present study.

This outcome in the case of a head-on collision is expected to be influenced by the collision angle of the droplets. In addition to the development of an asymmetric flow field, increasing the collision angle decreases the region of interaction of the droplet, which in turn decreases the collision energy responsible for the reflexive action. It can be seen in Fig. 6(b) that for $\theta=10.37^{\circ}$ the droplets coalesce after the collision and stretch in a direction inclined to the direction of impact. The resulting flow field is asymmetric with the recirculating regions appearing at the opposite sides of the ligament of the combined mass $[t=0.751 \mathrm{~ms}$ in Fig. $7(\mathrm{a}-\mathrm{ii})]$. Then, owing to the surface tension, the droplet contracts $(t=1.125 \mathrm{~ms})$ and then the inertia elongates it in the direction diagonally opposite to that of the initial stretching $(t=1.246 \mathrm{~ms})$. Due to the competition between the kinetic energy and surface energy, the droplet evolves into a tilted dumbbell-shaped droplet $(t=1.597 \mathrm{~ms})$. In this case, as the internal kinetic energy associated with fluid flow is insufficient to overcome the surface tension, the bulbous ends are pulled back, resulting in permanent coalescence. The droplet continues to exhibit shape oscillations (elongation and contraction) until the internal kinetic energy, which is responsible for these oscillations, completely dissipates in the form of viscous dissipation. Ashgriz and $\mathrm{Poo}^{3}$ found that for a low-viscosity liquid (e.g., water), permanent coalescence occurs when the effective kinetic energy is less than $75 \%$ of the surface energy; otherwise, it undergoes reflexive separation.

Increasing the collision angle further [Fig. 6(c) for $\theta=28.68^{\circ}$ ] decreases the interaction region significantly, and the major part of the mass associated with each droplet tends to move in the same impacting direction even after coalescing. Owing to the resulting shear, a dumbbell-shaped droplet with a thin ligament is formed at $t=0.510 \mathrm{~ms}$. It can be seen in Fig. 7 (a-iii) that, relative to $\theta=10.37^{\circ}$, the recirculating regions are closer to the fluid mass [also, see vorticity contours in Fig. 7(c)]. As in this case, the internal kinetic energy is able to overcome the effect of surface tension forces that try to contain the droplet as a single mass; the heavier masses at the two ends of the ligament are detached. From pressure field [Fig. 7(b)], we observe that the pressure field within the detached ligament is non-uniform. The curvature at neck connecting the ligament and bulbous ends causes the pressure in the ligament to be higher than that of the ambient due to the surface tension. Subsequently, the ligament also fragments into satellite droplets, as can be seen at $t=0.773 \mathrm{~ms}$ in Fig. 6(c). This collision outcome is termed "stretching separation" in the present study. We may, therefore, infer that increasing the collision angle for a fixed set of other parameters has a non-monotonic effect on the collision outcome, with the head-on collision and large collision angles resulting in separation, whereas permanent coalescence occurs at intermediate collision angles. We will come back to this point again later.

The fragmentation of the ligament and the detachment of satellite droplets occur via two mechanisms, namely, the end-pinching mechanism and capillary wave instability, ${ }^{37,38}$ which are operational in the cases of short and long ligaments, respectively. It can be seen that the breakup observed in the case of head-on collision [Fig. 6(a)] is due to the end-pinching mechanism, but the breakup for $\theta=28.68^{\circ}$ [Fig. 6(c)] is due to the capillary wave instability.

Next, in Fig. 8, we investigate the effect of velocity ratio, $V_{r}$ on the collision dynamics of two identical tetradecane droplets of size $R_{1}$ $=R_{2}=168 \mu \mathrm{m}$ undergoing oblique collision with $\theta=6.43^{\circ}$ in nitrogen at $1 \mathrm{~atm}$. The velocity of droplet 1 is fixed at $1.252 \mathrm{~m} / \mathrm{s}$ and velocity of droplet 2 is varied to obtain $V_{r}=0.8,1.15$, and 1.2. The temporal evolutions of the velocity, pressure, and vorticity fields for $V_{r}=0.8$, 1.15, and 1.2 are presented in Figs. 9(a)-9(c), respectively. Here, $W e=15.35, R e=149.6$. It can be seen that the collision outcome changes from permanent coalescence (for $V_{r}=0.8$ and 1.15) to reflexive separation (for $V_{r}=1.2$ ) by increasing the velocity ratio $\left(V_{r}\right)$. Increasing the impact velocity ratio between the droplets increases the shear, which in turn increases stretching of the combined liquid mass. Thus, it can be seen that the length of the filament with two blobs of liquid at its ends (see, the second row in Fig. 8) increases with increasing the velocity ratio. The associated flow fields have been depicted in the first row of Fig. 9(a). Then the surface tension tries to bring the droplet to a spherical shape, but the droplet gets elongated due to the inertia and the internal kinetic energy. Subsequently, for $V_{r}=1.2$, the central part of the ligament becomes thinner $(t=3.398 \mathrm{~ms})$ than that observed for low values of $V_{r}$, and the droplets breakup occurs due to capillary wave instability.

It can be seen in Figs. 9(a)-9(c) that the distributions of the flow, pressure, and vorticity fields and the recirculation region change according to the shape evolution of the droplet. Inspection of pressure 


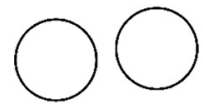<smiles>C1CCCCCCC1</smiles><smiles>C1CCCCCCC1</smiles>

$0.00 \mathrm{~ms}$

0.00

0.00
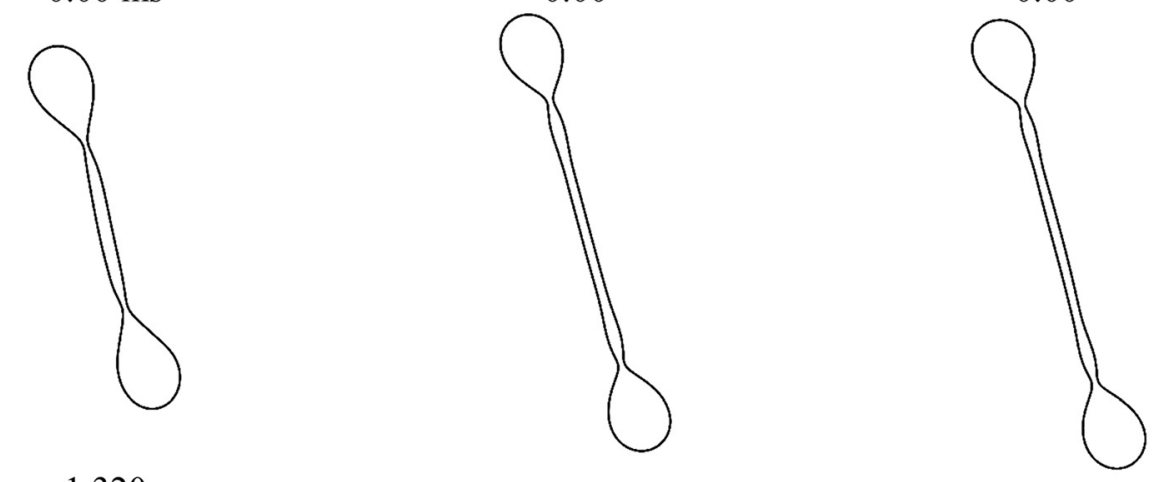

1.320

1.520

1.542

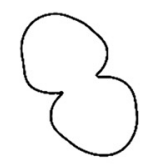<smiles>C1CCCCCCC1</smiles><smiles>C1CCCCCCCCC1</smiles>

2.223

2.618

2.553
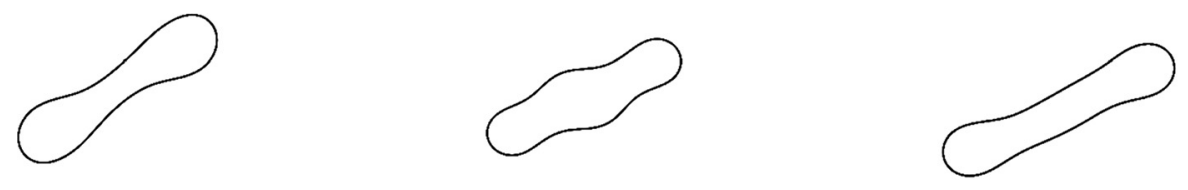

2.807

2.903

2.913
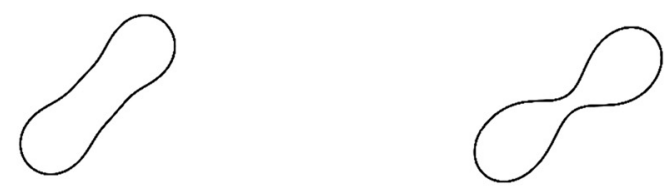

3.441

3.514

3.398
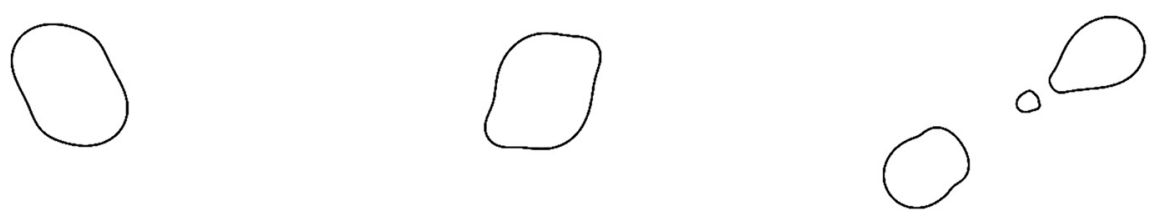

\subsection{0}

3.970

3.887

(a)

(b)

(c)

FIG. 8. Effect of the velocity ratio $\left(V_{r}=V_{2} / V_{1}\right)$ on the collision dynamics of two tetradecane droplets in nitrogen at 1 atm (a) $V_{r}=0.8$, (b) $V_{r}=1.15$, and (c) $V_{r}=1.2$. The other dimensional parameters are $R_{1}=R_{2}=168 \mu \mathrm{m}, V_{1}=1.252 \mathrm{~m} / \mathrm{s}$, and $\theta=6.43^{\circ}$. Thus, the values of the corresponding dimensionless numbers are We $=15.35$, $R e=149.6$, and $R_{r}=1$. 


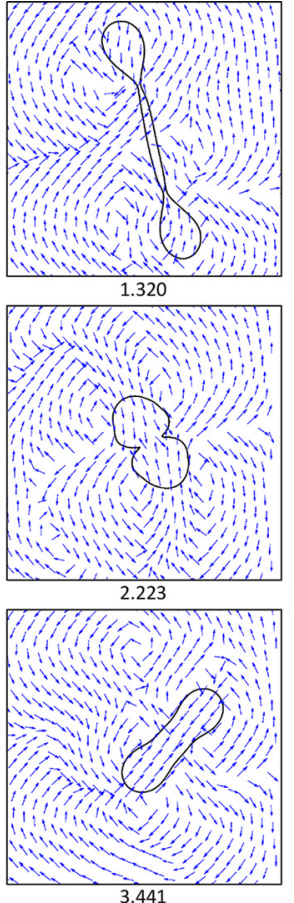

(i)
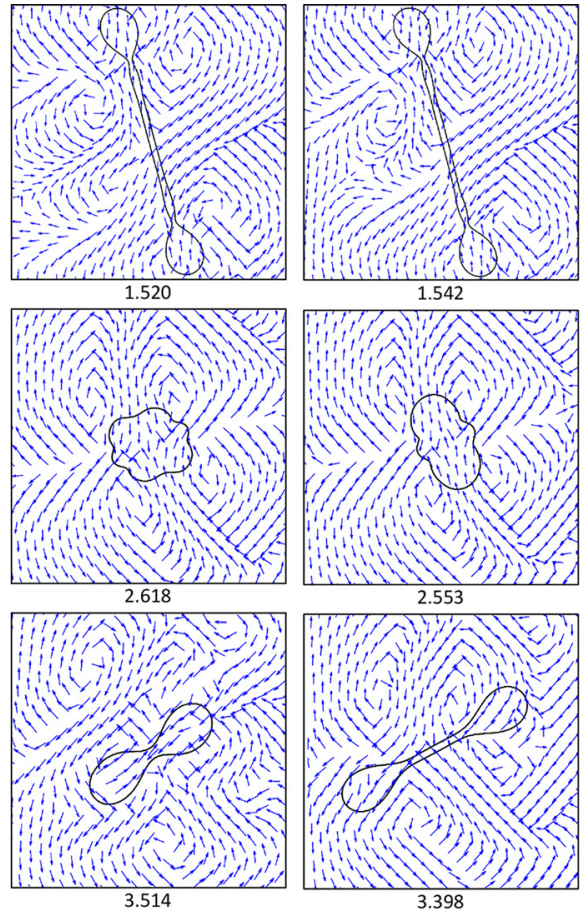

(ii)

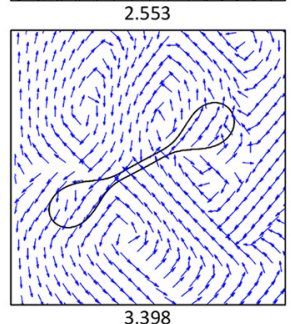

(iii)

(a)

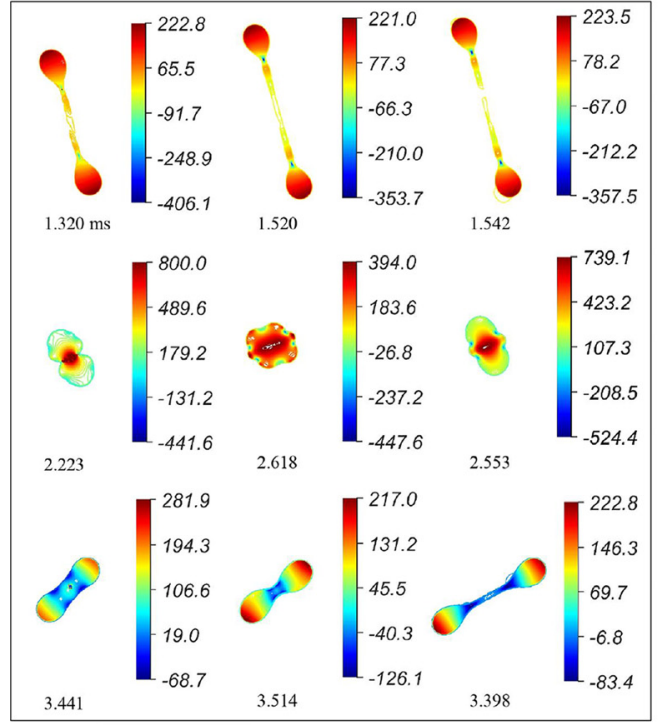

(i) (ii)

(b)

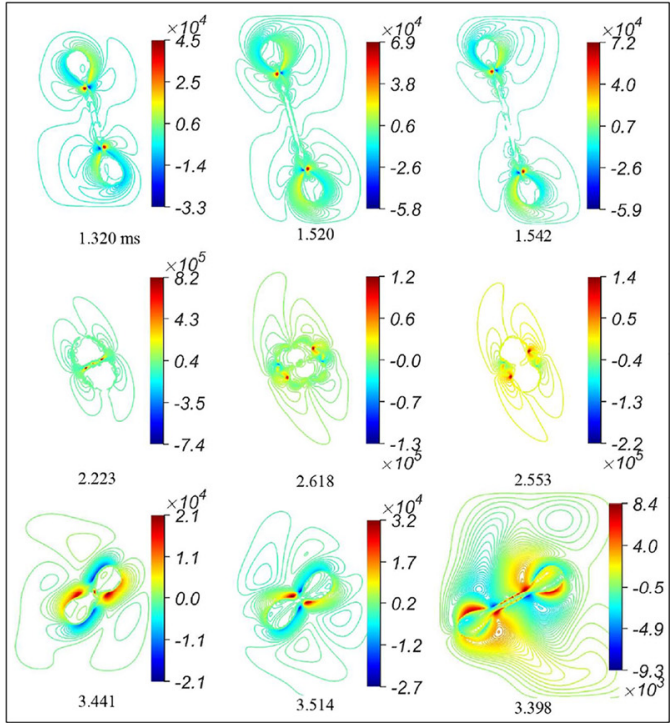

(i)

(ii)

(iii)

(c)

FIG. 9. Temporal evolution of (a) flow field, (b) pressure distribution, and (c) vorticity $(\partial v / \partial x-\partial u / \partial y)$ field for different values of velocity ratio $\left(V_{r}\right)$ in the case of collision of two tetradecane droplets in nitrogen at $1 \mathrm{~atm}$. In each panel, (i) $V_{r}=0.8$, (ii) $V_{r}=1.15$, and (iii) $V_{r}=1.2$. The other dimensional parameters are $R_{1}=R_{2}=168 \mu \mathrm{m}, V_{1}=$ $1.252 \mathrm{~m} / \mathrm{s}$, and $\theta=6.43^{\circ}$. Thus, the corresponding dimensionless numbers are $W e=15.35, R e=149.6$, and $R_{r}=1$. 
(a)

(b)

$\square$

$0.00 \mathrm{~ms}$<smiles>COCCO</smiles>

0.138<smiles></smiles>

0.242<smiles>C1CCCCC1</smiles>

0.420
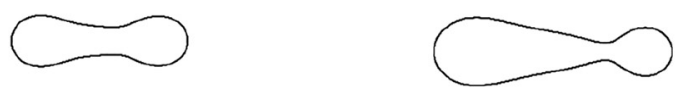

0.772

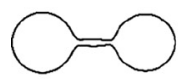

0.981

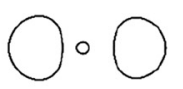<smiles>C1CCCCCC1</smiles><smiles>c1ccccc1</smiles><smiles>c1ccccc1</smiles>

0.00<smiles>C1CCCOCCC1</smiles>

0.170

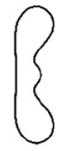

0.346

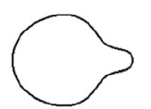

0.508

0.994

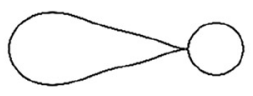

1.138 (c)

1.051

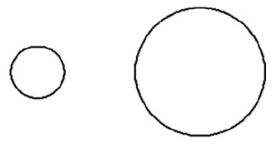

0.00<smiles>C1CCCCCCCCC1</smiles>

0.238

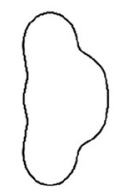

0.433

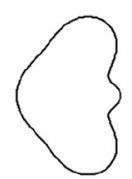

0.576

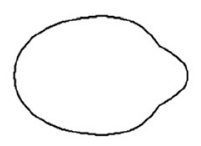

0.810

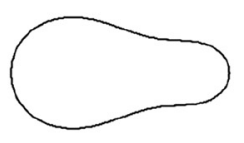

1.060

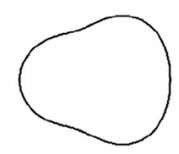

1.881
FIG. 10. Effect of the radius ratio $\left(R_{r}=\right.$ $R_{2} / R_{1}$ ) on the collision dynamics of two tetradecane droplets in nitrogen at $1 \mathrm{~atm}$ : (a) $R_{r}=1.2$, (b) $R_{r}=1.6$, and (c) $R_{r}=2.4$. The other dimensional parameters are $R_{1}=100 \mu \mathrm{m}, V_{1}=V_{2}=1.604$ $\mathrm{m} / \mathrm{s}$, and $\theta=0^{\circ}$. Thus, the corresponding dimensionless numbers are $W e=15$ $R e=114.27$, and $V_{r}=1$. 

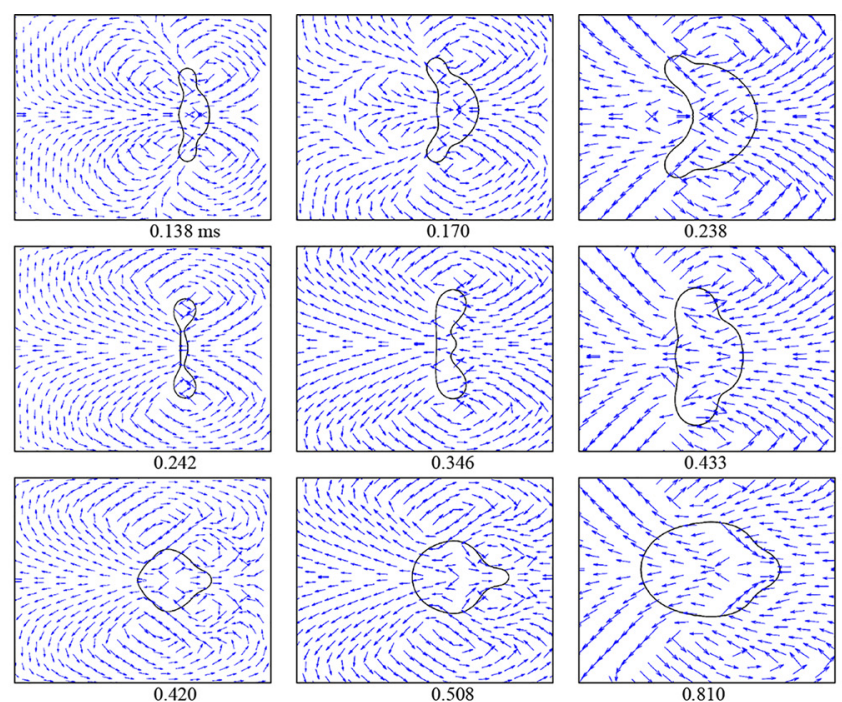

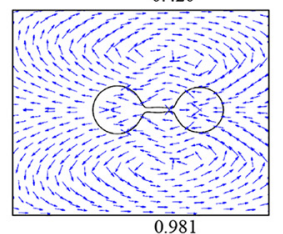

(i)

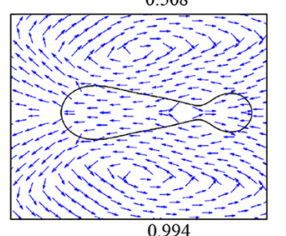

(ii)

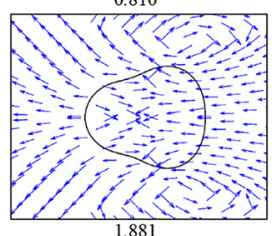

(iii)

(a)

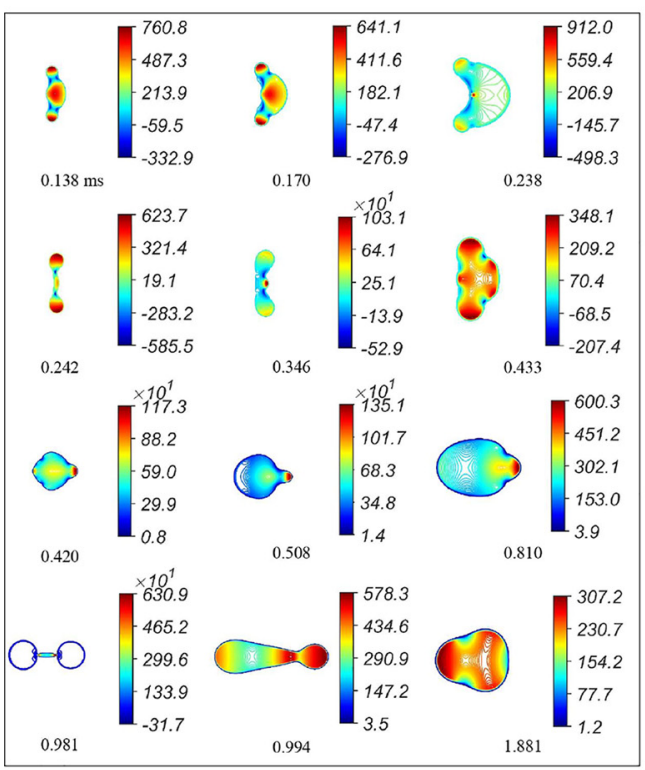

(i) (ii)

(b)

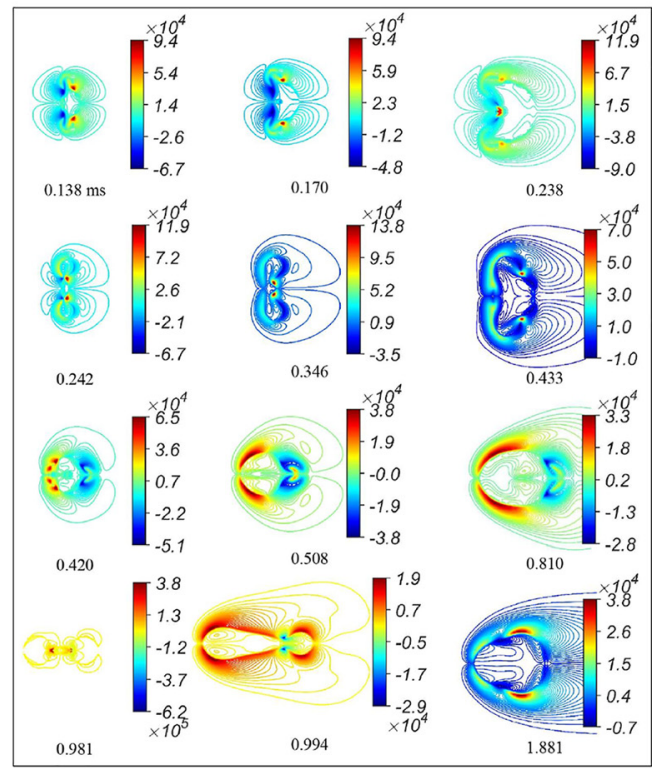

(i)

(ii)

(iii)

(c)

FIG. 11. Temporal evolution of (a) flow field, (b) pressure distribution, and (c) vorticity $(\partial v / \partial x-\partial u / \partial y)$ field for different values of radius ratio $\left(R_{r}\right)$ in the case of collision of two tetradecane droplets in nitrogen at $1 \mathrm{~atm}$. In each panel, (i) $R_{r}=1.2$, (ii) $R_{r}=1.6$, and (iii) $R_{r}=2.4$. The other dimensional parameters are $R_{1}=100 \mu \mathrm{m}, V_{1}=V_{2}=$ $1.604 \mathrm{~m} / \mathrm{s}$ and $\theta=0^{\circ}$. Thus, the corresponding dimensionless numbers are $W e=15, R e=114.27$, and $V_{r}=1$. 
distribution in Fig. 9(b) reveals that the pressure is maximum within blobs as the fluid is pushed through the connecting neck [first row in Fig. 9(b)] and after contraction, the central region of the contracted droplet which is sandwiched between two end caps [second row in Fig. 9(b)] has a pressure maximum and a low pressure at the connected surface segments which in turn causes deformation of droplets in a direction nearly perpendicular to the incoming flow from both sides of the capped portion of the droplets. In the third row of Fig. 9(b), it can be seen that the pressure at curved ends of the deformed droplet is high compared with the central portion. The droplets are separated if the kinetic energy is sufficient enough to overcome surface tension force, and subsequently, the bulbous ends of the deformed droplet pinch off from the neck as shown in Fig. 8(c). If the kinetic energy is insufficient to overcome the surface tension, the flow is simply directed toward the center of the drops [third row of Fig. 9(a) for $V_{r}=0.8$ and 1.15] as a result of the pressure differential. From vorticity contours in Fig. 9(c), it can be observed that the vorticity is higher in the second row of Fig. 9(c) than the first and third rows of Fig. 9(c). This indicates that the strength of vortices is minimum for a deformed droplet due to its minimum internal kinetic energy as compared to a contracted droplet of nearly spherical shape [second row of Fig. 9(c)]. It can also be seen that the sign of the vorticity is reversed after the deformation and contraction processes.

Then, to investigate the effect of the radius ratio, $R_{r}$ the radius of droplet 2 is varied while the radius of droplet 1 is fixed at $R_{1}=100$ $\mu \mathrm{m}$. In this case, the head-on collision $\left(\theta=0^{\circ}\right)$ of two tetradecane droplets with $V_{1}=V_{2}=1.604 \mathrm{~m} / \mathrm{s}$ in nitrogen at $1 \mathrm{~atm}$ is simulated, such that $W e=15$ and $R e=114.27$. Figures 10 (a) -10 (c) show the shape of the droplets before and after the collision for $R_{r}=1.2,1.6$, and 2.4, respectively. In the case of unequal-sized droplets, the capillary pressure associated with the small droplet is higher than that for the larger droplet, which influences the shape deformation of these droplets before and after the collision. At early times, it can be observed that the small droplet penetrates inside the larger droplet,

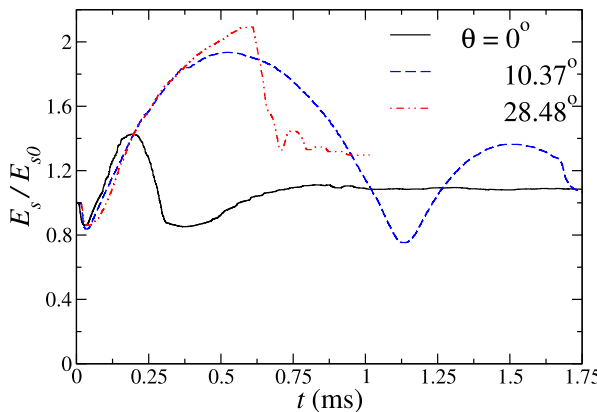

(a)

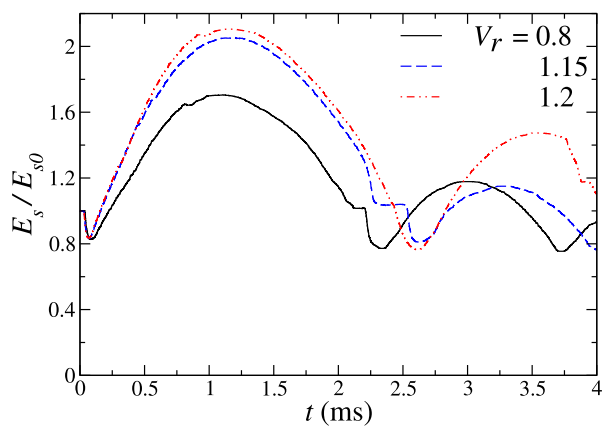

(c)

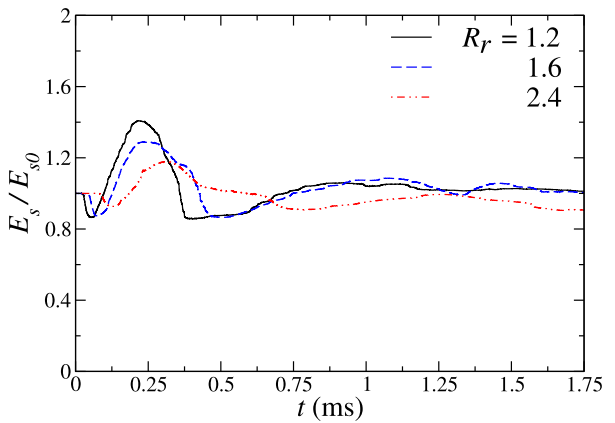

(e)

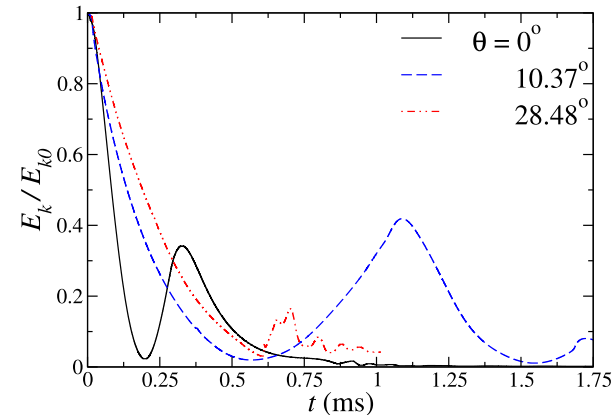

(b)

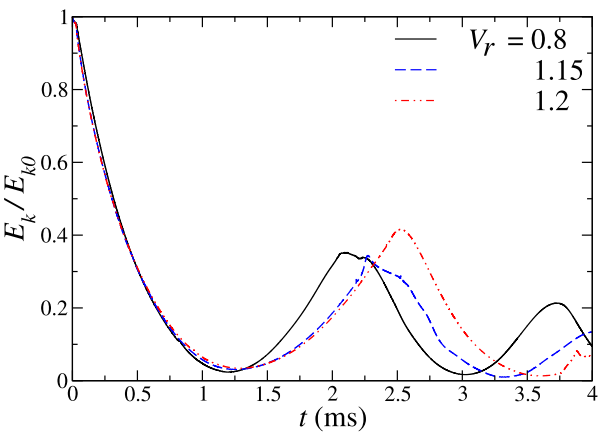

(d)

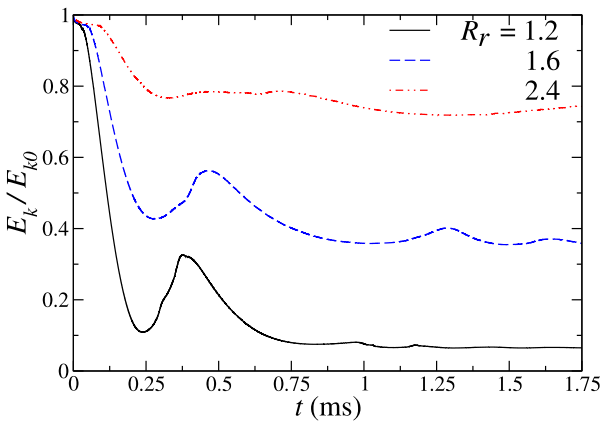

(f)
FIG. 12. Evolutions of the normalized surface energy $\left(E_{s} / E_{s 0}\right)$ and kinetic energy $\left(E_{k} / E_{k 0}\right)$ of droplets for different values of the $[(a)$ and (b)] collision angle (the parameters are the same as Fig. 6), [(c) and (d)] velocity ratio (the parameters are the same as Fig. 8), and [(e) and (f)] radius ratio (the parameters are the same as Fig. 10). The surface energy and kinetic energy are normalized by the initial surface and kinetic energy of the droplets. 


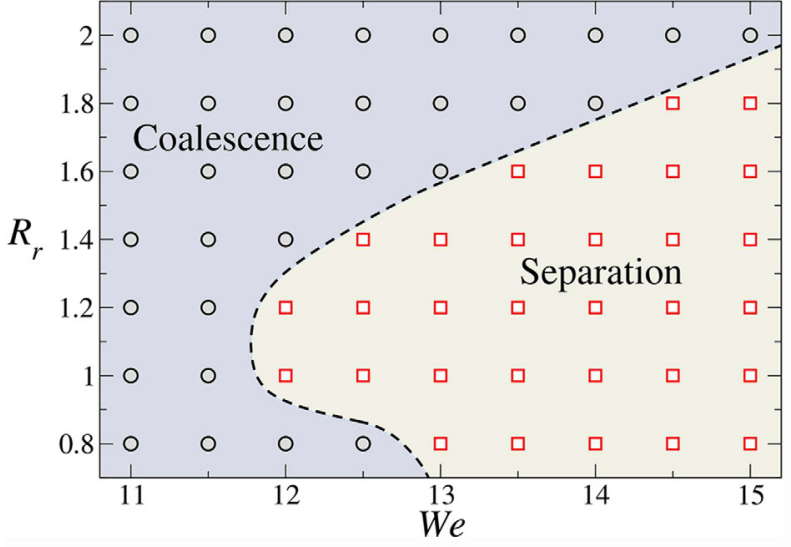

(a)

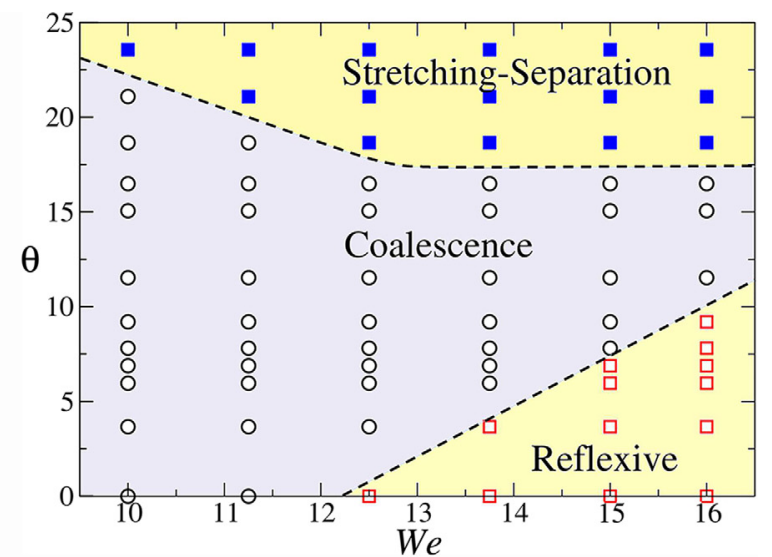

(b)

FIG. 13. Identification of different regimes for two tetradecane droplets in nitrogen at $1 \mathrm{~atm}$. (a) $\ln R_{r}-$ We space for $R_{1}=100 \mu \mathrm{m}, V_{r}=1$, and $\theta=0^{\circ}$. (b) In $\theta-W e$ space for $R_{r}=1\left(R_{1}=R_{2}=100 \mu \mathrm{m}\right)$ and $V_{r}=1$.

forming a mushroom-type shape (second row in Fig. 10). This creates a wake region in the side of the small droplet, which increases with increase in the radius ratio, $R_{r}$ [see the first row in Fig. 11(a)]. Subsequently, the combined mass of the droplets exhibits asymmetricshaped oscillations corroborated by the flow field (see Figs. 10 and 11). It can be seen that whereas the droplets with $R_{r}=1.2$ and 1.6 for this set of parameters undergo separation, and droplets with $R_{r}=2.4$ exhibit permanent coalescence. Hence, it can be inferred that an increase in the radius ratio suppresses the separation outcome. Tang et al. ${ }^{22}$ showed that in the case of two unequal-sized droplets undergoing head-on collision, the suppression of the separation outcome is due mainly to the increased viscous dissipation energy from the internal motion in the merged droplet before it starts stretching. Another observation is that for $R_{r}=1.2$ a satellite droplet is formed in addition to two primary droplets after breakup. This does not occur for the intermediate value of $R_{r}$. The formation of the satellite droplet when $R_{r}=1.2$ is due to the capillary instability of the liquid bridge connecting the two blobs of liquid mass, as shown in Fig. 10(a). For $R_{r}=1.6$ [Fig. 10(b)], the liquid bridge tends to stick to the larger droplet instead of forming a satellite droplet. Hence, the collision process in the case of unequal-sized droplets is significantly more complex than that for the identical droplets undergoing head-on collision.

In Figs. 12(a)-12(f), we have shown the temporal evolution of dimensionless surface $\left(E_{s} / E_{s 0}\right)$ and kinetic energy $\left(E_{k} / E_{k 0}\right)$ of the droplets for different values of the angle [Figs. 12(a) and 12(b)], velocity ratio [Figs. 12(c) and 12(d)], and radius ratio [Figs. 12(e) and 12(f)]. The surface energy and kinetic energy are normalized by the initial surface $\left(E_{s 0}\right)$ and kinetic energy $\left(E_{k 0}\right)$ of binary droplet system. It can be observed in Fig. 12 that during the initial deformation process, while the surface energy increases, the kinetic energy of the system decreases. The kinetic energy reaches a minimum value when the combined droplet mass deforms to its maximum. During contraction process as the deformed droplet contracts to retrieve its spherical shape, we observe that the kinetic energy increases until it reaches a certain maximum value, while the surface energy decreases to a minimum value. The conversion of the kinetic energy into surface energy and then surface energy into the kinetic energy continues during the entire collision process. In the case of collision resulting in coalescence, the competition between the surface energy and kinetic energy occurs for a longer duration as compared to collisions, resulting in separation. It can also be observed in Fig. 12 that the maximum (peak) value of the kinetic energy decreases with time due to viscous dissipation. As the combined droplet mass (in the case of coalescence) and the daughter droplets (in the case of separation) regain a spherical shape, the kinetic and surface energies become constant. This observation depicted in Fig. 12 is similar to that observed by Pan et al. ${ }^{19}$ and Cong et al. ${ }^{26}$

Finally, the regime maps showing different collision outcomes for two droplets impacting at the same velocities $\left(V_{r}=1\right)$ are presented in Figs. 13(a) and 13(b) on the $R_{r}-W e$ plane (for head-on collision and $R_{1}=100 \mu \mathrm{m}$ ) and the $\theta-W e$ plane (for $R_{1}=R_{2}=100 \mu \mathrm{m}$ ). It can be seen in Fig. 13(a) that in the case of a head-on collision, the droplets undergo permanent coalescence for low Weber numbers, but for high Weber numbers, the droplets display separation after temporary coalescence. The regime map on the $\theta-W e$ plane [Fig. 13(b)] reveals that for low Weber numbers, an increase in $\theta$ changes the collision outcome from permanent coalescence to stretching-separation. On the other hand, for high values of the Weber number, an increase in $\theta$ changes the collision outcome from reflexive-separation to coalescence to stretching-separation. It can be seen that the region associated with permanent coalescence shrinks as the Weber number is increased. The collision outcomes for the range of parameters considered in the present study are found to be consistent with the observation from earlier experimental studies of Tang et al. ${ }^{22}$ for head-on collision of unequal-sized droplets and Qian and $\operatorname{law}^{17}$ for oblique collision of identical droplets.

\section{CONCLUSIONS}

We investigated numerically the oblique collision of two unequal-sized liquid droplets in a gaseous environment in the framework of the coupled-level-set-volume-of-fluid (CLSVOF) approach, with particular attention to the study of the effect of the collision angle $(\theta)$, velocity ratio $\left(V_{r}\right)$, and radius ratio $\left(R_{r}\right)$ of the droplets. We validated our numerical solver against the previous experimental and 
numerical results associated with the head-on collision of two identical droplets. The collision outcomes (coalescence, bouncing, and separation) were found to be greatly influenced by the collision angle, velocity ratio, and radius ratio of the droplets. We observed that increasing the collision angle has a non-monotonic effect on the collision outcome; while permanent coalescence occurs at intermediate collision angles, head-on and large-angle collisions result in reflexive-separation and stretching-separation, respectively. Moreover, the breakup mechanisms of the ligament in head-on and large-angle collisions are also different. The end-pinching mechanism is operational in the case of the head-on collision, as also reported in previous studies. ${ }^{25,37,38} \mathrm{On}$ the other hand, the capillary wave instability is found to be responsible for the ligament breakup for large collision angles.

We found that for low velocity ratios, the droplets coalesce permanently, but for high velocity ratios, the combined liquid mass after temporary coalescence undergoes stretching due to shear to form a long ligament that subsequently breaks up. Increasing the radius ratio was found to suppress the separation outcome due to the increase in viscous dissipation, as also observed by Tang et al. ${ }^{22}$ Finally, by conducting a large number of simulations, we presented the collision outcomes and the boundary separating them on $R_{r}-W e$ and $\theta-W e$ planes.

\section{ACKNOWLEDGMENTS}

One of the authors (G.B.) acknowledges his gratitude to J. C. Bose National Fellowship of SERB, Government of India.

\section{DATA AVAILABILITY}

The data that support the findings of this study are available from the corresponding author upon reasonable request.

\section{REFERENCES}

${ }^{1}$ G. M. Faeth, "Current status of droplet and liquid combustion," in Energy and Combustion Science (Elsevier, 1979), pp. 149-182.

${ }^{2}$ R. Murugan, P. S. Kolhe, and K. C. Sahu, "A combined experimental and computational study of flow-blurring atomization in a twin-fluid atomizer," Eur. J. Mech., B 84, 528-541 (2020).

${ }^{3}$ N. Ashgriz and J. Y. Poo, "Coalescence and separation in binary collisions of liquid drops,” J. Fluid Mech. 221, 183-204 (1990).

${ }^{4}$ J. R. Adam, N. R. Lindblad, and C. D. Hendricks, "The collision, coalescence, and disruption of water droplets," J. Appl. Phys. 39(11), 5173-5180 (1968).

${ }^{5}$ M. Agrawal, A. R. Premlata, M. K. Tripathi, B. Karri, and K. C. Sahu, "Nonspherical liquid droplet falling in air," Phys. Rev. E 95, 033111 (2017).

${ }^{6} \mathrm{M}$. Balla, M. K. Tripathi, and K. C. Sahu, "Shape oscillations of a nonspherical water droplet,” Phys. Rev. E 99(2), 023107 (2019).

${ }^{7}$ M. Agrawal, R. K. Katiyar, B. Karri, and K. C. Sahu, "Experimental investigation of a nonspherical water droplet falling in air," Phys. Fluids 32(11), 112105 (2020).

${ }^{8}$ S. T. Thoroddsen and K. Takehara, "The coalescence cascade of a drop," Phys. Fluids 12(6), 1265-1267 (2000).

${ }^{9}$ H. Deka, G. Biswas, K. C. Sahu, Y. Kulkarni, and A. Dalal, "Coalescence dynamics of a compound drop on a deep liquid pool,” J. Fluid Mech. 866, R2 (2019).

${ }^{10} \mathrm{X}$. Chen, S. Mandre, and J. J. Feng, "Partial coalescence between a drop and a liquid-liquid interface," Phys. Fluids 18(5), 051705 (2006).

${ }^{17} \mathrm{~F}$. Blanchette and T. P. Bigioni, "Partial coalescence of drops at liquid interfaces," Nat. Phys. 2(4), 254-257 (2006).

${ }^{12}$ F. Blanchette and T. P. Bigioni, "Dynamics of drop coalescence at fluid interfaces,” J. Fluid Mech. 620, 333 (2009).
${ }^{13}$ B. Ray, G. Biswas, and A. Sharma, "Generation of secondary droplets in coalescence of a drop at a liquid-liquid interface,” J. Fluid Mech. 655, 72-104 (2010).

${ }^{14}$ P. K. Kirar, K. Alvarenga, P. Kolhe, G. Biswas, and K. C. Sahu, "Coalescence of drops on the free-surface of a liquid pool at elevated temperatures," Phys. Fluids 32(5), 052103 (2020).

${ }^{15}$ G. D. M. MacKay and S. G. Mason, "The gravity approach and coalescence of fluid drops at liquid interfaces," Can. J. Chem. Eng. 41(5), 203-212 (1963).

${ }^{16}$ F. H. Zhang, E. Q. Li, and S. T. Thoroddsen, "Satellite formation during coalescence of unequal size drops,” Phys. Rev. Lett. 102(10), 104502 (2009).

${ }^{17}$ J. Qian and C. K. Law, "Regimes of coalescence and separation in droplet collision," J. Fluid Mech. 331, 59-80 (1997).

${ }^{18}$ J. Eggers, J. R. Lister, and H. A. Stone, “Coalescence of liquid drops," J. Fluid Mech. 401, 293-310 (1999).

${ }^{19}$ K. L. Pan, C. K. Law, and B. Zhou, "Experimental and mechanistic description of merging and bouncing in head-on binary droplet collision," J. Appl. Phys. 103(6), 064901 (2008).

${ }^{20} \mathrm{~S}$. Unverdi and G. Tryggvason, "A front-tracking method for viscous, incompressible, multi-fluid flows,” J. Comput. Phys. 100(1), 25-37 (1992).

${ }^{21}$ M. R. Nobari, Y.-J. Jan, and G. Tryggvason, "Head-on collision of drops-A numerical investigation,” Phys. Fluids 8(1), 29-42 (1996).

${ }^{22}$ C. Tang, P. Zhang, and C. K. Law, "Bouncing, coalescence, and separation in head-on collision of unequal-size droplets," Phys. Fluids 24(2), 022101 (2012).

${ }^{23}$ M. Kumar, R. Bhardwaj, and K. C. Sahu, "Coalescence dynamics of a droplet on a sessile droplet," Phys. Fluids 32(1), 012104 (2020).

${ }^{24}$ N. Nikolopoulos, G. Strotos, K. S. Nikas, and G. Bergeles, "The effect of weber number on the central binary collision outcome between unequal-sized droplets," Int. J. Heat Mass Transfer 55(7-8), 2137-2150 (2012).

${ }^{25}$ H. Deka, G. Biswas, S. Chakraborty, and A. Dalal, "Coalescence dynamics of unequal sized drops," Phys. Fluids 31(1), 012105 (2019).

${ }^{26} \mathrm{H}$. Cong, L. Qian, Y. Wang, and J. Lin, "Numerical simulation of the collision behaviors of binary unequal-sized droplets at high weber number," Phys. Fluids 32(10), 103307 (2020).

${ }^{27}$ N. Goyal, J. Shaikh, and A. Sharma, "Bubble entrapment during head-on binary collision with large deformation of unequal-sized tetradecane droplets," Phys. Fluids 32(12), 122114 (2020).

${ }^{28}$ J. U. Brackbill, D. B. Kothe, and C. Zemach, “A continuum method for modeling surface tension,” J. Comput. Phys. 100, 335-354 (1992).

${ }^{29}$ D. Gerlach, G. Tomar, G. Biswas, and F. Durst, "Comparison of volume-offluid methods for surface tension-dominant two-phase flows," Int. J. Heat Mass Transfer 49(3-4), 740-754 (2006).

${ }^{30} \mathrm{~F}$. H. Harlow and J. E. Welch, "Numerical calculation of time-dependent viscous incompressible flow of fluid with free surface," Phys. Fluids 8(12), 2182-2189 (1965).

${ }^{31} \mathrm{X}$. D. Liu, S. Osher, and T. Chan, "Weighted essentially non-oscillatory schemes," J. Comput. Phys. 115(1), 200-212 (1994).

${ }^{32}$ Y. C. Chang, T. Hou, B. Merriman, and S. Osher, "A level set formulation of Eulerian interface capturing methods for incompressible fluid flows," J. Comput. Phys. 124(2), 449-464 (1996).

${ }^{33}$ E. G. Puckett, A. S. Almgren, J. B. Bell, D. L. Marcus, and W. J. Rider, "A highorder projection method for tracking fluid interfaces in variable density incompressible flows," J. Comput. Phys. 130(2), 269-282 (1997).

${ }^{34}$ M. Sussman and E. G. Puckett, "A coupled level set and volume-of-fluid method for computing $3 \mathrm{~d}$ and axisymmetric incompressible two-phase flows," J. Comput. Phys. 162(2), 301-337 (2000).

${ }^{35} \mathrm{G}$. Son and N. Hur, "A coupled level set and volume-of-fluid method for the buoyancy-driven motion of fluid particles," Numer. Heat Transfer, Part B 42(6), 523-542 (2002).

${ }^{36} \mathrm{G}$. Son, "Efficient implementation of a coupled level-set and volume-of-fluid method for three-dimensional incompressible two-phase flows," Numer. Heat Transfer, Part B 43(6), 549-565 (2003).

${ }^{37}$ H. A. Stone and L. G. Leal, "Relaxation and breakup of an initially extended drop in an otherwise quiescent fluid," J. Fluid Mech. 198, 399-427 (1989).

${ }^{38}$ H. A. Stone, B. J. Bentley, and L. G. Leal, "An experimental study of transient effects in the breakup of viscous drops,” J. Fluid Mech. 173, 131-158 (1986). 